\title{
Range and regression effects in magnitude scaling
}

\author{
ROBERT TEGHTSOONIAN and MARTHA TEGHTSOONIAN \\ Smith College, Northampton, Massachusetts 01063
}

\begin{abstract}
The relation between power law exponents obtained by magnitude estimation and mag. nitude production was studied for both loudness and perceived distance. While the results confirm the usual finding of higher values for production for relatively large stimulus ranges, just the opposite occurs when the stimulus range is short, necessitating a revision of the Stevens-Greenbaum regression principle. The relation between range and exponent was explored, both for the case in which several intensities are presented for judgment and for the simpler case of only two intensities. In both cases, a power relation was described relating stimulus ratios to judgmental ratios, with exponents containing both range-dependent and range-independent components.
\end{abstract}

In the study of psychophysical judgment, a central issue has concerned the relation between stimulus intensity and judgments of perceived magnitude. While much work has focused on the form of the psychophysical law relating these variables (see, for example, Stevens, 1975), Helson (1947) and those who followed in his tradition have been concerned with the contextual constraints operating on the observers' judgments. Thus, while the loudness of a signal is obviously influenced by its sound-pressure level, Cross (1973) has shown that it is also influenced by the intensity of the preceding signal. And, more generally, Anderson (1975) and Parducci, Calfee, Marshall, and Davidson (1960) have shown that the judgment of a given signal may be influenced by the properties of the entire set being judged. The present report is concerned with one such property-the range of intensities presented for judgment-and its effect on the psychophysical power law.

The power law exponents reported by S. S. Stevens and his associates for 24 perceptual continua have been shown by R. Teghtsoonian (1971) to be almost entirely predictable by the stimulus range employed: the correlation between the obtained exponents, $n$, and the reciprocal of log stimulus range (the ratio of greatest to smallest stimulus intensity) was +.94 , implying that the judgmental range (estimated from the fitted power function) is nearly constant.

The experimental work on which this paper is based was supported by Grant BMS-73-06944 from NSF. The preparation of the manuscript was supported by Grant NS 12597 from NINDS, and was carried out in part while $M$. Teghtsoonian held an NRC-NAS Resident Research associateship at the U.S. Army Natick Research and Development Command. We thank Ann Horn and Susan Crow for assistance in running subjects and analyzing data. Requests for reprints should be sent to Robert Teghtsoonian, Clark Science Center, Smith College, Northampton, Massachusetts 01063.
Teghtsoonian proposed that this result could follow from two assumptions: first, that the stimulus ranges selected by Stevens and his associates define a nearly constant proportion of dynamic range (ratio of the greatest to smallest stimulus magnitudes to which the observer is sensitive and responsive); second, that under matching instructions the dynamic range for any one continuum can be exhaustively mapped into the dynamic range for any other continuum. Given these assumptions, it follows that the dynamic ranges for all perceptual continua are subjectively equal, and that, therefore, variation in exponent simply reflects variation in dynamic range.

But Stevens' data are also compatible with a possibility suggested by Poulton (1968) that an oberver's judgmental range is constant, regardless of the range of intensities presented. From such a point of view, the exponent of the psychophysical power relation contains no useful information, since it simply relates the invariant judgmental range provided by an insensitive observer to the stimulus range arbitrarily selected by the experimenter. This latter view holds the exponent to be nothing more than a redundancy for the selected stimulus range. Teghtsoonian's argument, on the other hand, is that the exponent serves as an index (and perhaps the most easily obtained measurement) of a characteristic of the observer that is of fundamental biological significance -his range of sensitivity.

In a subsequent paper, Teghtsoonian (1973) argued that if the invariant judgmental range hypothesis were correct, then varying the stimulus range within any given continuum (intramodal range variation) should have the same effect on the exponent as the range variation across several different continua, the effect seen in the analysis of Stevens' studies (intermodal range variation); he summarized the available 
evidence showing that this is not the case and included a brief account of a loudness scaling experiment in which stimulus range was systematically varied. One purpose of the present report is to provide a more complete account of that loudness scaling experiment and to report a formally identical investigation of perceived distance. For both continua, stimulus values were presented for magnitude estimation in four conditions defining different stimulus ranges, from $1 / 4$ to $2 \log$ units. The concern in both cases was to determine the relation between exponent and stimulus range, and to compare that relation with the simple inverse proportionality that occurs when range variation is intermodal.

The second purpose of this report is to compare two explanations of what has been called the "regression effect" in psychophysical scaling. Stevens and Greenbaum (1966) noted that the exponent obtained for a pair of continua in a matching experiment depends on which is controlled by the observer and which by the experimenter. They summarized a body of evidence showing magnitude estimation to yield lower exponents than magnitude production and argued, as have many writers since then, that observers appear to restrict the range of stimuli that they control: in magnitude estimation, this restriction applies to the variable on the ordinate, whereas in magnitude production, it is the variable plotted on the abscissa that is so affected. The result, it is argued, is to diminish exponents below their unbiased, true value, in one case, or to inflate them, in the other. Recent analyses (e.g., Cross, 1973) have sought to provide exact models for the extent of this bias, but continue to assume that estimation exponents will necessarily be smaller than production exponents.

A different explanation has been offered by R. Teghtsoonian (1973). He suggested that observers may behave as if avoiding judgments that, in relation to the preceding judgment, define extremely large or small ratios. The rationale for this hypothesis is most easily developed in the simplest case of two stimulus intensities, $\phi_{1}$ and $\phi_{2}$, defining a ratio, $r_{\phi}$, and the corresponding judgments, $J_{1}$ and $J_{2}$, defining a judgmental ratio, $\mathrm{r}_{\mathrm{J}}$. The simple form of the psychophysical power law,

$$
\mathbf{J}=\mathbf{k} \phi^{\mathbf{n}}
$$

leads to the prediction that

$$
\log r_{J}=n \log r_{\phi} .
$$

Teghtsoonian, summarizing evidence from a number of studies, found that the relation between $\log r_{\mathbf{J}}$ and $\log \mathrm{r}_{\phi}$ is, indeed, linear but that an additive constant is required, so that

$$
\log r_{J}=n^{\prime} \log r_{\phi}+c .
$$

This finding led him to propose that the principal variables in the psychophysical power relation may be stimulus and response ratios, $\mathrm{r}_{\phi}$ and $\mathrm{r}_{\mathrm{J}}$, rather than the absolute quantities, $\phi$ and $\mathrm{J}$, as Stevens had implied. A similar conclusion concerning the importance of stimulus and response ratios has been argued by Krantz (1972), but it is fair to say that such a view is not universally shared.

If Equation 3 is correct, and if $\mathrm{c}$ is a positive value (as reported in Teghtsoonian, 1973), then an estimate of $\mathbf{n}$ for magnitude estimation that is calculated as $\log r_{J} / \log r_{\phi}$, will yield a value of $n$ that represents the sum of two components, $n^{\prime}+\left(c / \log r_{\phi}\right)$, which decreases with increases in $r_{\phi}$. This is the intramodal range effect already described. Next, if the result for magnitude production follows the general case of Equation 3, it will be

$$
\log r_{\phi}=m \log r_{N}+c,
$$

where $r_{N}$ is the ratio of two assigned numbers and $r_{\phi}$ is the ratio defined by the corresponding setting made by the observer on the continuum under his control. Again, if the exponent is estimated by $\log r_{N} / \log r_{\phi}$, as suggested by Equation 1, the result according to Equation 4 will be the reciprocal of $\mathrm{m}+\left(\mathrm{c} / \log \mathrm{r}_{\mathrm{N}}\right)$, and will therefore increase with $\mathrm{r}_{\mathrm{N}}$ (and $r_{\phi}$ ), just opposite to the result for magnitude estimation. Thus, the relation between estimation and production exponents cannot be stated absolutely, for it depends on the range of stimuli employed. Evidence supporting these predictions, including a two-stimulus magnitude production experiment for loudness, modeled on the previously cited estimation experiment, was reported by Teghtsoonian (1973). The present report includes a more detailed account of that study as well as a new study of apparent distance modeled on the loudness experiment.

In the first study, the two continua used were loudness and number. For magnitude estimation, the subject matched numbers to target loudnesses; for magnitude production, he matched loudnesses to target numbers. There were four ranges of values on the target continua: a magnitude estimation subject was asked to match numbers to loudnesses covering a .25-log-unit range, a .50-log-unit range, a 1.0-logunit range, or a 2.0-log-unit range; similarly, a magnitude production subject matched loudnesses to numbers covering a range of $.25, .50,1.0$, or $2.0 \mathrm{log}$ units. In addition, within each range, the first two stimuli presented were selected to define a constant logarithmic interval $(.15, .30, .60$, or $1.2 \log$ units) for each subject. In the second study, the two continua used were distance and number. The design was the same, but there were only three ranges of values for the target continuum $(.25, .50$, or $1.0 \log$ unit) and the separation of the first two stimuli $(.15, .30$, or .60 log unit). The magnitude estimation and mag- 
nitude production procedures were designed to be the same except for the identities of the target and matching continua; both used a free-modulus procedure in which no standard is designated and no modulus assigned.

\section{SOUND INTENSITY}

Method
Subjects
The subjects were Smith College women undergraduates, age
17 to 22 , volunteers who received course credit in an introductory
psychology course for their participation. $N=16$ in each of four
groups for magnitude estimation and for magnitude production.
See Results for the criterion for retaining subjects.

\section{Apparatus}

A pure tone signal of $3,000 \mathrm{~Hz}$ was produced by a HewlettPackard Model 241A oscillator and presented to the subject binaurally through a pair of PDR-8 earphones. Stimulus duration was controlled by a Grason-Stadler Model 829E electronic switch.

\section{Procedure}

The magnitude estimation and magnitude production procedures were designed to be, as nearly as possible, identical: in one case, the subject matched number to sound intensity; in the other, she matched sound intensity to number, but otherwise the procedure was the same.

Magnitude estimation: Number matched to sound intensity. There were four groups, defined by the total voltage range covered by the stimuli presented, $.25, .50,1.0$, or $2.0 \log$ units $(5,10,20$, or $40 \mathrm{~dB}$ SPL). Within each range, there were six stimulus values, at equal logarithmic intervals of $.05, .10, .20$, and $.40 \mathrm{log}$ units $(1,2,4$, and $8 \mathrm{~dB})$, respectively, for the four groups. The midpoint of each stimulus range was $84 \mathrm{~dB} \mathrm{SPL}$, and the tone was $3,000 \mathrm{~Hz}$.

The six stimulus values were presented twice in a random order, with the following restrictions: the first stimulus could not be the least or most intense, and the first two stimuli presented defined a range of $.15, .30, .60$, or $1.2 \log$ units $(3,6,12$, or $24 \mathrm{~dB}$ ), respectively, for the four groups.

A free-modulus procedure, in which there is no designated standard or assigned modulus, was used. The instructions told the subject to assign numbers to represent the relative loudnesses of the tones she was to hear. She was told that the ratios of successive loudnesses might sometimes be very large or very small. Each tone was presented three times, for $2.5 \mathrm{sec}$ each, with an intertone interval of $2.5 \mathrm{sec}$.

Magnitude production: Sound intensity matched to number. There were four groups, defined by the total range of numbers presented, .25, .50, 1.0, or 2.0 log units. Each group received six numbers spaced at equal logarithmic intervals $(.05, .10, .20$, and $.40 \log$ units, respectively, for the four groups). The midpoint of the stimulus range was not determined in advance.

For each group, the first stimulus presented was selected from a designated set of four numbers. The subject selected a sound intensity to match the number. Depending upon the value she selected, and according to a predetermined schedule, a set of five other numbers at the appropriate intervals were selected to be the remaining stimulus values. The second stimulus number presented defined an interval of $.15, .30, .60$, or $1.2 \log$ units relative to the first stimulus number. The remaining four stimulus values were presented in random order, then the six were presented a second time in a different random order.

This procedure is the analog of free-modulus magnitude estimation, as the first value presented varies across subjects and the subject is free to make any match to the first presented number. (In practice, a subject occasionally made an initial match that would have required her on subsequent matches to set intensities greater or less than the equipment permitted; in these cases, the initial match was discarded and the subject asked to make a softer or louder setting.) This freedom from the constraint of a designated standard or prescribed modulus meant, however, that the identical stimulus numbers could not be presented to each subject: different numbers were used, although within each group the ratios between numbers were constant. This meant that subjects in the shortest-range group might have received numbers between 2.5 and 4.5 , at one extreme, and between 7.1 and 13, at the other, while subjects in the longest-range group might have received numbers between .0056 and .56 or between $\$ 6$ and 5,600 .

The instructions told the subject that she was to adjust the loudness of a tone so that it corresponded to the size of the numbers she was to hear. She was told that the ratio of successive numbers might sometimes be very large or very small. The experimenter presented the stimuli by saying the number aloud. The subject then adjusted the signal, a $3,000-\mathrm{Hz}$ tone that alternated $2.5 \mathrm{sec}$ on with $2.5 \mathrm{sec}$ off, until she was satisfied with the match. On the first trial, the adjustment started from a setting of maximum attenuation; on subsequent trials, the adjustment started with the signal at the setting made on the previous trial.

\section{The Full-Range Analysis \\ Results}

Data for individual subjects were fitted by a method of least squares: for magnitude estimation, $\log$ geometric means of the two numbers assigned each stimulus intensity were regressed on the log adjusted voltage values; for magnitude production, log geometric means of the two adjusted voltage values assigned each stimulus number were regressed on the $\log$ numbers. The subjects for whom the coefficient of determination, $\mathrm{r}^{2}$, was less than .80 were discarded and replaced; for .25-, .50-, 1.0-, and 2.0-log-unit groups, respectively, it was necessary to run $24,18,17$, and 16 subjects to obtain 16 who met the criterion value for $\mathbf{r}^{2}$ for magnitude estimation, and $17,16,16$, and 17 for magnitude production. ${ }^{1}$ The least squares fits accounted for a mean of $92 \%$ of the variance in $\log$ responses for magnitude estimation and $97 \%$ for magnitude production, indicating that power functions are adequate fits to the individual judgments. The exponents of these individual power functions were subjected to KruskalWallis rank-order analyses of variance. For both magnitude estimation $(\mathrm{H}=11.22, \mathrm{p}<.05)$ and magnitude production $(\mathrm{H}=15.97, \mathrm{p}<.05)$, there were significant differences among ranges. Subsequent Mann-Whitney U tests indicated that (1) for magnitude estimation, the median exponents for the four ranges differed significantly ( $\mathrm{p}<.05$, one-tailed test) except for .25 vs. $.50, .50$ vs. 1.0 , and 1.0 vs. 2.0 , and (2) for magnitude production, the métian exponents differed significantly $(p<.05$, one-tailed test) except for .25 vs. .50 and .50 vs. 1.0 .

The open circles in Figure 1A show these exponents and illustrate the significant range effect-for both estimation and production, the larger the range of 


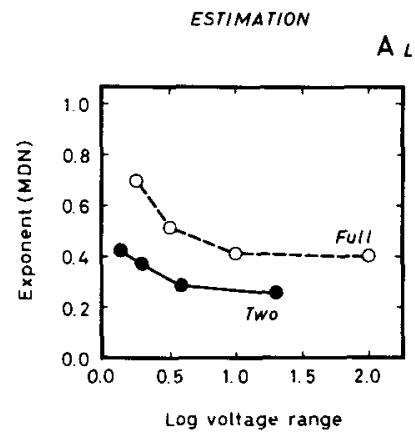

A Loudness

PRODUCTION

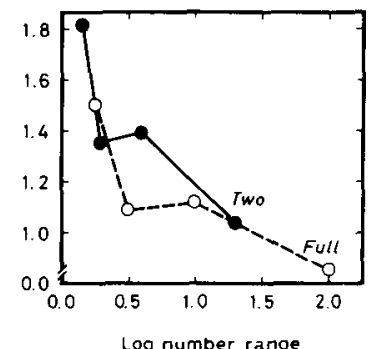

B Distance
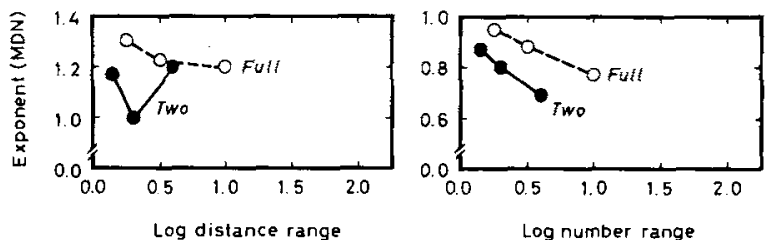

Figure 1. Exponent as a function of range of presented stimulus values in estimation (left column) and production (right column) for two-stimulus (filled circles) and full-range (open circles) analyses. (Note the differences in absolute values between ordinates in left and right columns. For $A, N=16$ in each of eight groups. For $B, N=12$ in each of six groups.)

presented stimuli, the lower the exponent. R. Teghtsoonian (1973) has cited these magnitude estimation data to show that the intramodal range effect obtained is much less than the intermodal effect he previously reported (1971). ${ }^{2}$ Although the change in exponent is greater for magnitude production, it also falls far short of the change produced by intermodal range. The magnitude production data thus confirm Teghtsoonian's conclusion that the intermodal range effect cannot be attributed to the subject's adjusting his fixed response range to span whatever range of stimulus values is presented by the experimenter.

A direct comparison of the magnitude estimation and magnitude production data is facilitated by expressing number as a function of voltage in both cases, even though number is the independent variable for magnitude production. The exponents for magnitude production were subjected to a reciprocal transformation (since, if Voltage $=a$ Number ${ }^{n}$, then Number $=a^{\prime}$ Voltage $\left.^{1 / n}\right)$, and these values are shown at the left of Figure $2 A$. As the range of voltages $\left(R_{V}\right)$ increases, magnitude estimation exponents decrease, while magnitude production exponents increase, apparently at a more rapid rate.

At each value of $R_{V}$, the difference between estimation and production exponents is what Stevens and Greenbaum (1966) called the regression effect: the subject produces a different exponent, depending on which continuum he controls. Mann-Whitney
$U$ tests of the difference between median estimation and production exponents for each range (although note that $R_{V}$ is not exactly equated for each comparison) showed that, for 1.0- and 2.0-log-unit-range groups, the differences were significant $(p<.05$, two-tailed test). These results demonstrate that the regression effect is range-dependent, being larger for large values of $R_{V}$ and decreasing as $R_{V}$ decreases. Extrapolation of the magnitude production function shows the regression effect eventually reversing in direction for $\mathrm{R}_{\mathrm{V}}$ less than about $.35 \log$ units. $^{3}$ Whether the magnitude production or magnitude estimation exponent is larger apparently depends on stimulus range. This result contradicts the Stevens and Greenbaum (1966) prediction that production exponents will always be larger than estimation exponents, and thus casts doubt on their explanation of the regression effect as due to the subjects' restricting the range of values on the response continuum.

The data may also be expressed by plotting the range of numbers $\left(R_{N}\right)$ (produced or presented) as a function of the range of voltages $\left(R_{V}\right)$ (presented or produced), as in Figure 3A. The presented range was determined by the experimenter; the produced range is estimated from the median exponent $\mathrm{n}$ by $\mathrm{n}=$ $\log R_{N} / \log R_{V}$, where $\log R_{N}$ is estimated for magnitude estimation and $\log R_{V}$ for magnitude production. Figure $3 \mathrm{~A}$ shows that the linearity between log stimulus range and log judgmental range assumed by Stevens' law is supported, but that the
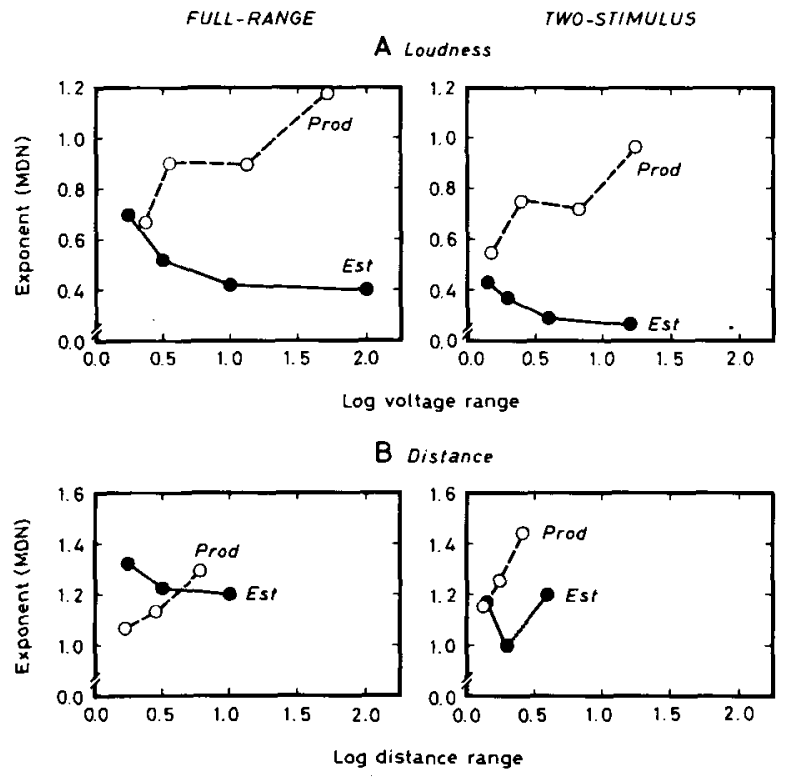

Figure 2. Exponent of the power function relating number to the physical continuum for voltage and distance. Full range data are to the left; two-stimulus, to the right. Filled circles show estimation; open circles, production. For $A, N=16$ in each of eight groups. For $B, N=12$ in each of six groups. 


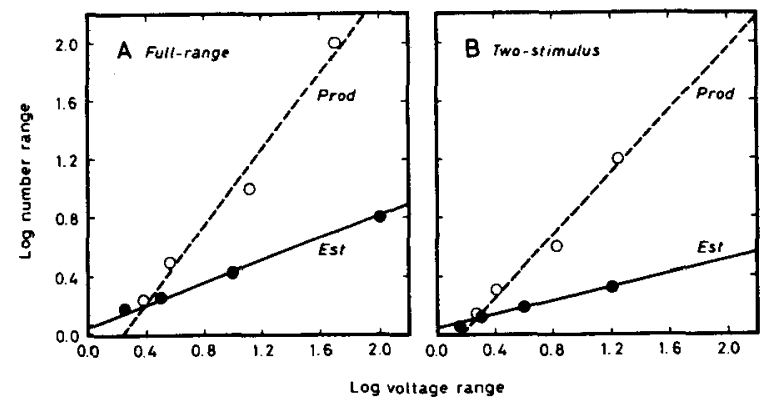

Figure 3. Number range as a function of voltage range. Both variables are scaled logarithmically. Number was produced by the subject in estimation (filled circles), provided by the experimenter in production (open circles), $\mathbf{N}=16$ in each of eight groups. For $A$, estimation, $\log \mathbf{R}_{\mathrm{N}}=.36 \log \mathbf{R}_{\mathrm{V}}+.08$; production, $\log R_{N}=1.31 \log R_{V}-.30$. For $B$, estimation, $\log r_{N}=.23$ $\log r_{v}+.04 ;$ production, $\log r_{N}=1.06 \log r_{V}-.17$.

assumption of a zero intercept is not. The results of intramodal stimulus range variation are best described as

$$
\log R_{N}=m \log R_{V}+k,
$$

where the introduction of the additive constant, $k$, to summarize the effect of range, makes it possible to preserve the slope constant, $\mathrm{m}$, as an invariant, independent of stimulus range.4 For magnitude estimation, the least squares fit $\left(r^{2}=.999\right)$ yields $\mathrm{m}=.36, \mathrm{k}=.08$; for magnitude production, $\mathrm{m}=$ 1.31 and $k=-.30$ (the least squares fit was to the inverse function, with $\mathrm{m}=.76$ and $\mathrm{r}^{2}=.976$ ). R. Teghtsoonian (1973) has cited the magnitude estimation data previously; the magnitude production data confirm the existence of a nonzero intercept.

\section{The Two-Stimulus Analysis}

The order of stimulus presentation was arranged so that for each full-range group, the first two stimulus values defined a constant range-for the .25-logunit group, the first two stimuli were $.15 \mathrm{log}$ units apart; for the .50-log-unit group, .30 log units; for the 1.0-log-unit group, .60 log units; for the 2.0-logunit group, $1.2 \mathrm{log}$ units. Thus the presentation of the first two stimulus values constituted a smaller experiment within the full-range experiment. These data were analyzed in the same way as the full-range data, with exponents determined by expressing the $\log$ ratio of the first two responses as a proportion of the log ratio of the first two stimuli. The median exponent for each range is shown by the filled circles in Figure 1A.

For magnitude estimation, the curve for the twostimulus exponent has the same form as that for the full-range exponent, but the two-stimulus exponents have values about $60 \%$ smaller at the same ranges of voltage. These differences among two-stimulus exponents, however, are not reliable (Kruskal-Wallis analysis of variance showed $\mathrm{H}=0.69, \mathrm{p}>.05$ ). For magnitude production, the two-stimulus curve lies close to the full-range curve. Reliable differences do exist among these values; a Kruskal-Wallis analysis of variance $(H=15.00, p<.05)$ and subsequent $\mathrm{U}$ tests $(\mathrm{U}<83, \mathrm{p}<.05$, one-tailed test) showed that all pairwise comparisons yielded significant differences except for .25 vs. .50 and .50 vs. 1.0 .

The exponent can be calculated for number, presented or produced, as a function of voltage, produced or presented; the right-hand portion of Figure 2A shows these values. ${ }^{3}$ For groups with the same target ranges, production exponents differ reliably from estimation exponents in all comparisons except that for the .25-log-unit groups (Mann-Whitney $\mathrm{U}$ tests showed $\mathrm{U}<75, \mathrm{p}<.05$, two-tailed test, for $.50,1.0$, and $2.0 \log$ units). Comparison with the lefthand portion of Figure $2 \mathrm{~A}$ reveals the close similarity between full-range and two-stimulus data in the relationship between exponent and range of voltages. In Figure 3B, number range is shown as a function of voltage range; comparison with Figure $3 \mathrm{~A}$, for the full-range, reveals the similarity between full-range and two-stimulus data. For estimation, the fitted function is $\log r_{N}=.23 \log r_{V}+.04$, and for production, $\log r_{N}=1.06 \log r_{V}-.17$; in both cases, the estimate of a range-free exponent ( $n^{\prime}$ in Equation 3) is smaller for the two-stimulus experiment.

To sum up, the two-stimulus data show the same general pattern of relationships as do the full-range data: increasing stimulus ranges produce decreasing exponents, and the relation between production and estimation exponents depends on range, with the difference least at the shortest range.

\section{DISTANCE}

\section{Method}

\section{Subjects}

The subjects were Smith College women undergraduates, aged 17 to 22 , volunteers who received course credit in an introductory psychology course for their participation. $N=12$ in each of the three groups for magnitude estimation and for magnitude production.

\section{Apparatus}

The experiment took place in a room, $12.1 \times 3.5 \mathrm{~m}$. The subject sat at one end of the room. There were tables, chairs, bookcases, and miscellaneous items along the walls. The room was lit by fluorescent strip lamps in the ceiling. On the floor, running from the subjects' feet to the far wall was a single line of HO-gauge railroad track; on the track was a toy train locomotive with a white $3 \times 5$ in. index card mounted on the end of the locomotive nearest the subject, in her frontal-parallel plane. A control switch allowed the subject to move the locomotive in either direction during the magnitude production procedure. Distance was measured from a point on the floor directly below the subject's eyes to a point directly below the bottom edge of the index card.

\section{Procedure}

The procedure for the distance experiments was formally 
identical to that for the sound intensity experiments, except that only three stimulus ranges were used.

Magnitude estimation: Number matched to distance. There were three groups, defined by the total distance range, $.25, .50$, or $1.0 \log$ unit. The midpoint of each range was $3.7 \mathrm{~m}$. Within each range, there were six stimulus values, at equal logarithmic intervals $(.05, .10$, or $.20 \mathrm{log}$ units); the actual distance ranges were $2.77-4.93,2.08-6.58$, and $1.17-11.7 \mathrm{~m}$, respectively.

Each of the six stimulus values was presented once, then again, in a random order, except that the first two stimuli defined a constant range within each group- $.15, .30$, and $.60 \mathrm{log}$ units, respectively-and neither the nearest nor the furthest distances could occur as the first stimulus. A free-modulus procedure, with no designated standard or assigned modulus, was used.

The instructions told the subject to assume a "standard" position, sitting with head held in line with a marker at her left. She was to assign numbers to represent how far away the train appeared. The subject averted her head while the experimenter changed the train's position; she looked at the train only when making her judgment.

Magnitude production: Distance matched to number. There were three groups, defined by the total range of numbers presented, $.25, .50$, or $1.0 \log$ unit. The numbers presented for each group were determined exactly as described for magnitude production of sound intensity. The first two stimuli defined a constant range within each group, $.15, .30$, or .60 log units.

The instructions told the subject to assume the "standard" position. She was to adjust the location of a target-an index card on a toy train-so that its distance matched the presented numbers. She was told that the ratio of successive numbers might sometimes be very large or very small. She was cautioned not to count floor tiles as the basis for her judgments. She adjusted the location of the target, by means of a switch on the arm of her chair, until she was satisfied with the match. On the first trial, initial distance of the target was zero. On subsequent trials, the adjustment was made starting with the target at its previous location. The subject could not see the target between trials.

\section{The Full-Range Analysis}

\section{Results}

Data were analyzed in the same way as for the sound-intensity experiments. Subjects who did not meet the criterion of $\mathrm{r}^{2} \geqslant .80$ were discarded and additional subjects were run to obtain $\mathrm{N}=12$. None was discarded in the magnitude estimation procedure, one was discarded from the .25 -log-unit group, and one was discarded from the 1.0-log-unit group in the magnitude production procedure. The least squares fits accounted for a mean of $96 \%$ of the variance in log individual responses for magnitude estimation and $\mathbf{9 8 \%}$ for magnitude production, indicating that power functions are good fits to the individual judgments.

Figure $1 \mathrm{~B}$ shows the exponent for each range for magnitude estimation and production. These data agree with those for sound intensity in showing the exponent to be range-dependent. The decrease in exponent with range for magnitude estimation, although in the expected direction, was not significant according to a Kruskal-Wallis analysis of variance $(H=1.18, p>.05)$. As with sound intensity, the magnitude production exponents showed a larger range effect; this was significant $(H=7.79, p<.05)$, and subsequent Mann-Whitney tests showed that both .25- and .50-log-unit ranges had higher exponents than the 1.0-log-unit range $(U<42, p<.05$, one-tailed test). Künnapas (1960) has suggested that distance exponents are range-dependent, and these data confirm his suggestion. They extend to indoor settings the range effect reported in an outdoor setting by Teghtsoonian and Teghtsoonian (1970). The absolute magnitude of the range effect is greater for sound intensity than for distance.

Figure 2B shows the exponent for each range for both estimation and production when number is expressed as a function of distance in both cases, even though number is the independent variable for magnitude production. For every group except magnitude production at the .25 -log-unit range, either 11 of 12 or all 12 subjects had exponents greater than 1.0. Thus, as Teghtsoonian and Teghtsoonian (1969) have reported, the exponent for indoor distance is greater than 1.0.

Not only do these data confirm those for sound intensity, showing that the regression effect is rangedependent, but also, for distance ranges less than about four to one (.6 log units), that the production exponent is lower than the estimation exponent. The crossover of the two functions suggested by the sound intensity data is seen clearly here. For the same target ranges, Mann-Whitney tests showed that the magnitude production exponent is lower than the magnitude estimation exponent for $.25 \log$ units $(\mathrm{U}=37, \mathrm{p}=.05$, two-tailed test) and no different for .50 and $1.0(\mathrm{U}>37, \mathrm{p}>.05$, two-tailed test).

Figure $4 A$ shows the range of numbers, $R_{N}$ (produced or presented), as a function of the range of distances, $R_{D}$ (presented or produced). The presented range was determined by the experimenter;

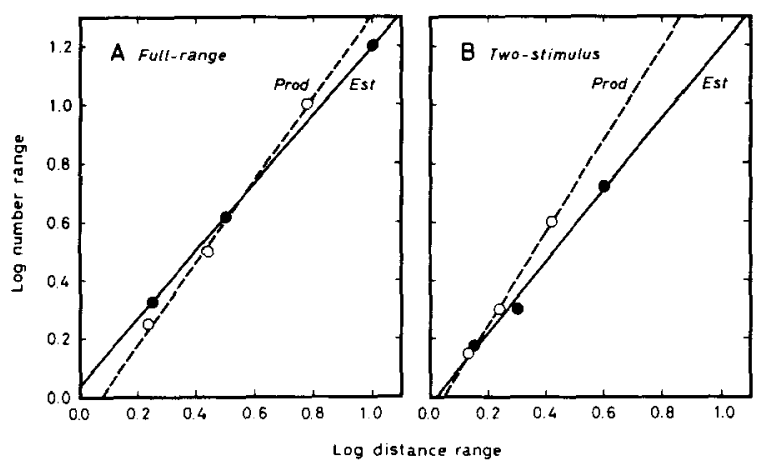

Figure 4. Number range as a function of distance range. Both variables are scaled logarithmically. Number was produced by the subject in estimation (filled circles), presented by the experimenter in production (open circles). $N=12$ in each of six groups. For $A$ estimation, $\log \mathbf{R}_{\mathrm{N}}=1.17 \log \mathbf{R}_{\mathrm{D}}+.03 ;$ production, $\log \mathbf{R}_{\mathrm{N}}=$ $1.41 \log R_{D}-.10$. For $B$, estimation, $\log r_{N}=1.24 \log r_{D}-.03$; production, $\log r_{N}=1.58 \log r_{D}-.07$. 
the produced range is estimated from the median exponent $n$, by $n=\log R_{N} / \log R_{D}$. As with the sound-intensity data, the results of intramodal stimulus variation are well described as a linear relation with a nonzero intercept between $\log$ stimulus range and log judgmental range. The slope constant has the value 1.17 for magnitude estimation and 1.41 for magnitude production; the intercept is .03 for estimation, -.07 for production.

\section{The Two-Stimulus Analysis}

The data for the first two stimuli presented, which define a constant range within each group, were analyzed separately, in the same way as the full-range data. For each subject, the range of numbers and the range of distances defined by two stimuli and two responses were used to obtain a median exponent, which is shown by the filled circles in Figure 1B. For magnitude estimation, the two-stimulus exponent did not decrease monotonically with an increase in stimulus range; for magnitude production, it did. Neither set of differences was significant, however, according to Kruskal-Wallis analyses of variance $(\mathrm{H}>5.99, \mathrm{p}>.05)$. The right-hand portion of Figure 2B shows exponent when number (produced or presented) is taken as a function of distance (produced or presented). Figure 4B shows number range as a function of distance range: for magnitude estimation (filled circles), the least squares fitted line has a slope of 1.24 and an intercept of -.03 ; for production (open circles), 1.58 and -.07 .

In general, the data from the two-stimulus analysis for distance show the same pattern as for the fullrange analysis: increasing stimulus ranges produce decreasing exponents, and the relation between production and estimation exponents depends on range, with the production exponent smaller than the estimation exponent at the shortest range. However, the strength of this conclusion is tempered both by the lack of statistical significance of the differences and by the occurrence of an anomalously large value for the magnitude estimation group with range $0.6 \log$ units.

\section{Discussion}

It is clear from both loudness and distance judgments that intramodal range of stimulation does exert a systematic effect on the exponent of Stevens' law. At least two features of this effect merit comment. First, it takes a different form than the effect associated with the change in continuum under study, although, for magnitude estimation, both entail a decrease in exponent with increasing stimulus range. The intramodal effect found in this study may best be described by Equation 3, showing a linear relation between estimated log judgmental range and log stimulus range with a nonzero additive constant.
In this case, the variation in stimulus range is entirely due to the experimenter. The intermodal effect, on the other hand, consists of a simple reciprocal relation between exponent and log stimulus range, where the latter is thought to estimate dynamic range and is a value constrained by the structure of the nervous system. There is, thus, no justification for the practice of assessing range effects by indiscriminately tabling together data resulting from both intramodal and intermodal range variation effects.

Second, the intramodal effect is evident not only in the conventional multistimulus experiment where the measured stimulus range may never be defined for the observer by any two successive stimulus presentations, but, perhaps more fundamentally, in a simple two-stimulus experiment requiring just two judgments from the observer. Indeed, it can be shown that the form and magnitude of the former is derivable from the result for the two-stimulus experiment on the assumption that performance in a multistimulus experiment is regarded as the outcome of successive applications of Equation 3 to successive pairs of stimuli (see appendix).

The dependence of exponent on stimulus range, whether shown in the full-range analysis or in the two-stimulus analysis, adds to the already large body of evidence that the result of no single experiment can be accepted as defining a "true" exponent. It is clear that measured exponents are governed by a host of parameters, of which range is just one, and the important task is to provide a quantitative description of those parametric effects. Such a description is provided for intramodal range variation by Equation 3, or by its antilog equivalent,

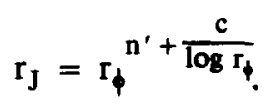

'It is important to note that the quantity $n^{\prime}$ in this expression is invariant over all values of $r_{\phi}$, whereas the exponent $n$, calculated for any given experiment, will be $n^{\prime}+c / \log r_{\phi}$, including not only $n^{\prime}$ but, as an additive quantity, the range-dependent term $c / \log r_{\phi}$. Thus, the empirical determination of Equation 3 makes it possible to identify a power law exponent that is independent of the range employed in any particular experiment. In this sense, Equation 6 can be regarded as an improvement on Equation 1. It should be emphasized that the discrepancy between Stevens' law (Equation 1 or Equation 2) and the suggested revision (Equation 3 or Equation 6) is not just that the former assumes a small value of the constant $c$, whereas the latter proposes a large value. Stevens' law makes no provision for a range effect, so the exponent $\mathrm{n}$ will differ for every range used; Equation 3 permits the measured and variable exponent $\mathrm{n}$ to be partitioned into a range-independent exponent $n^{\prime}$ and a range-dependent factor $c / \log r_{\phi}$. 
The present results confirm the existence of the regression effect. In previous work, while the magnitude estimation procedure was often free-modulus, most magnitude production procedures imposed a modulus; here we used a free-modulus technique for both estimation and production, but the differences in exponent persisted despite the equivalent procedures. The results also require a modification of the usual interpretation of this regression effect. It is evident from the present results that both estimation and production methods entail characteristic biases: neither $n$ nor $1 / \mathrm{m}$ are bias-free estimates of what might be termed $\beta$. But it is also apparent that the direction of these biases is not as usually stated because $n$ is not always less than $1 / \mathrm{m}$; at small values of $r_{\phi}$, the relation may be reversed. ${ }^{6}$ Such a result is consistent with the simple revision of Stevens and Greenbaum's (1966) argument noted in the introduction, namely that observers tend to avoid extreme judgmental ratios, with the outcome illustrated in Figures 3 and 4.

It is interesting to relate these findings to the analysis offered recently by Cross (1973) of order effects obtained by him (Cross, 1973; Cross \& Rotkin, 1975) as well as Luce and Green (1974) and Ward (1973). Those studies have shown a tendency for the judgment of a given stimulus to be higher when the preceding stimulus is greater than that being judged and lower when the preceding stimulus is smaller than that being judged, what has been called a tendency toward assimilation. Since, according to this argument, intense stimuli are more likely to be preceded by weaker ones, and weak stimuli by stronger ones, the necessary result is a regressive tendency of judgments toward a mean value. In fact, Cross (1973) has provided an elegant model for these sequential effects that permits quantification of this source of bias.

However, the present results imply that assimilation is not the only possible outcome in sequences of number-matching tasks. For stimulus intervals defining small ratios, the tendency to avoid small judgmental ratios may take the form of a contrast effect: when the preceding stimulus is only slightly smaller than the present one, the judgment of the latter is inflated. Although no such contrast effects have been reported in the literature, the present analysis argues that this is due to the range of stimuli employed. Given the structure of Cross' model, it will be easy enough to test the prediction that either contrast or assimilation may characterize order effects in psychophysical judgment, and that the size of the interstimulus interval determines which occurs.

In conclusion, some advice may be welcomed by investigators whose primary interest is not methodology but perhaps the study of some particular sensory system, and whose only interest in range effects is how to avoid them. Few such investigators will (or should) care to perform the parametric investigation necessary to estimate $\mathrm{n}^{\prime}$, the rangeindependent exponent of Equations 3 and 6 . It is, however, clear from the form of that function that the larger the value of $r_{\phi}$ employed in any particular experiment, the smaller will be the quantity $c / \log r_{\phi}$, and the closer therefore will be the obtained value of $n$ to the quantity $n^{\prime}$. In simple terms: when it is impractical to employ more than one value of $r_{\phi}$, use the largest value that is consistent with the safety and comfort of the observer. (Although this principle has been observed in some laboratories, no precise rationale for it has been previously stated.) It should be added that by relying on wide-range exponents, investigators can expect better agreement in their estimates, but this is not the same as a biasfree estimate. As Figure 2 made clear, there can be a substantial difference between production and estimation exponents for all but a single range value. But, since the location of this crossover point may depend on as yet unidentified parameters, it seems premature to advise any particular stimulus range as the key to a bias-free exponent. In the meantime, the advice to prefer wide-range exponents is intended for those who, while waiting for a theoretical truth to emerge, would like a simple improvement in reliability. A corollary to this principle states: when faced with conflicting values of $n$ in the literature, prefer the estimate obtained with the larger range. If experimenters make an effort to employ larger ranges, one result must be a reduction in the variability of $n$ due to range effects.

\section{APPENDIX}

Briefly, the derivation of the intramodal range effect in a multistimulus experiment from a two-stimulus, twojudgment experiment can be summarized as follows.'

For a pair of stimulus intensities defining the ratio $r_{J}$, the corresponding judgments define a ratio $r_{\mathrm{J}}$, such that

$$
\log r_{J}=n \log r_{\phi}+c .
$$

But Equation 7 was established for $r_{\phi}>1$ regardless of whether the greater of the two intensities occurred first or second. If $r_{\phi}$ is defined as the second intensity divided by the first, then, when $r_{\phi}<1$, Equation 7 becomes

$$
\log r_{\mathbf{J}}=\mathbf{n} \log r_{\phi}-c
$$

or, more generally and without restrictions on the size of $r_{\phi}$,

$$
\log r_{J}=n \log r_{\phi}+c \frac{\log r_{\phi}}{\left|\log r_{\phi}\right|}
$$

Assume, in the more usual multistimulus experiment in which several intensities are presented for judgment, that beginning with some arbitrary judgment made for the 
initial stimulus, the relation of the judgment for each subsequent stimulus to that for its predecessor is described by Equation 9. If, further, all stimulus sequences are explored, the problem is to calculate a mean value for $\log \mathrm{J}_{\mathbf{i}}$, where $\mathrm{J}_{\mathrm{i}}$ is the judged value for a particular stimulus intensity, $\Phi_{i}$.

Assume $\mathrm{N}$ values of $\Phi$, evenly spaced on a $\log$ scale, with an interval, $M$, between successive values of $\log \Phi$. Let $P$ be the value of the judgment for the initial stimulus in the sequence, regardless of its identity, and assume that the judged value for succeeding stimuli in the sequence can be determined by repeated applications of Equation 9. It can be shown that, as a result,

$$
E\left(J_{i}\right)=P\left(\frac{\Phi_{i}}{\pi_{\phi}}\right)^{n+\frac{2 c}{M N}}
$$

That is, the expected judged value of a stimulus can be obtained by expressing the intensity of that stimulus as a ratio of the geometric mean of all $\mathrm{N}$ intensities, raising it to the power $n+(2 \mathrm{c} / \mathrm{MN})$, and multiplying by the arbitrary scale factor, P. Of course, this closely resembles Stevens' law, but the exponent for Equation 10 includes the rangedependent term, MN.

If Equation 10 is written for $\Phi_{\mathrm{T}}$ and $\Phi_{\mathrm{B}}$, the top and bottom values in the set of $\mathrm{N}$ intensities, then the corresponding judgments, $J_{T}$ and $J_{B}$, define a ratio, $R_{J}$, which can be described as a function of $R_{\phi}=\left(\Phi_{\mathrm{T}} / \Phi_{\mathrm{B}}\right)$ :

$$
\mathbf{R}_{\mathbf{J}}=\mathbf{R}_{\phi}^{\mathbf{n}+\frac{2 c}{\mathrm{MN}}}
$$

Taking logs of both sides yields

$$
\log R_{J}=n \log R_{\phi}+2 c \frac{N-1}{N}
$$

since $\log \mathbf{R}_{\phi}=\mathbf{M}(\mathrm{N}-1)$.

It should be noted that for $N=2$, Equation 12 is identical to Equation 7, as required. Further, for any value of $\mathrm{N}$, Equation 12 is equivalent to Equation 5 (describing the multistimulus loudness estimation results), with the additive constant $k$ of Equation 5 corresponding to $2 c[(N-1) / N]$ in Equation 6. Indeed, it is striking to recall that $k$ was found to be .08 and $c$ (the additive constant in the two-stimulus analysis) was .04 .

\section{REFERENCES}

ANDERson, N. On the role of context effects in psychophysical judgment. Psychological Review, 1975, 82, 462-482.

Cross, D. V. Sequential dependencies and regression in psychophysical judgments. Perception \& Psychophysics, 1973, 14, 547-552.

Cross, D. V., \& Rotkin, L. The relation between size and apparent heaviness. Perception \& Psychophysics, 1975, 18, 79-87.

HeLson, H. Adaptation-level as frame of reference for prediction of psychophysical data. American Journal of Psychology, 1947, 60, 1-29.

KRANTZ, D. H. A theory of magnitude estimation and crossmodality matching. Joumal of Mathematical Psychology, 1972, 9, 168-199.
KÜNNAPAS, T. Scales for subjective distance. Scandinavian Journal of Psychology, 1960, 1, 187-192.

LuCE, R. D., \& GREen, D. M. The response ratio hypothesis for magnitude estimation. Journal of Mathematical Psychology, 1974, 11, 1-14.

Parducci, A., Calfee, R., Marshall, L., \& Davidson, L. Context effects in judgment: Adaptation level as a function of the mean, midpoint, and median of the stimuli. Journal of Experimentai Psychology, 1960, 60, 65-77.

Poulton, E. C. The new psychophysics: Six models for magnitude estimation. Psychological Bulletin, 1968, 69, 1-19.

Stevens, S. S. Psychophysics: Introduction to its perceptual, neural and social prospects. New York: Wiley, 1975.

STEVens, S. S., \& GREenbaum, H. Regression effect in psychophysical judgment. Perception \& Psychophysics, 1966, 1, 439-446.

Teghtsoonian, R. On the exponents in Stevens' law and the constant in Ekman's law. Psychological Review, 1971, 78, 71-80.

Teghtsoonian, R. Range effects in psychophysical scaling and a revision of Stevens' law. American Journal of Psychology, 1973, 86, 3.27.

Tegrtsoonian, M., \& Teghtsoonian, R. Scaling apparent distance in natural indoor settings. Psychonomic Science, 1969, 16, 281-283.

Teghtsoonian, R., \& Teghtsoontan, M. Scaling apparent distance in a natural outdoor setting. Psychonomic Science, 1970, 21, 215-216.

WARD, L. M. Repeated magnitude estimations with a variable standard: Sequential effects and other properties. Perception \& Psychophysics, 1973, 13, 193-200.

\section{NOTES}

1. In retrospect, it seems unwise to have specified $r^{2}$ as a criterion for retaining subjects in a study of range effects. Since $r^{2}$ is statistically sensitive to range effects and is also monotonically related to the slope of the regression line, it is clear that to discard subjects with low values of $r^{2}$ is to invite a systematic bias in the resulting estimates of the exponent. (However, it should also be noted that the slope of the regression line is less sensitive than $r^{2}$ to changes in range.) To check the extent of this bias in the present results, a separate analysis was made for the first 16 subjects run in each of the four groups, irrespective of obtained values of $r^{2}$. The results were virtually identical with those reported for the selected groups. When range is to be an experimental variable, the standard error of estimate may be a more appropriate goodness-of-fit index than $r^{2}$.

2. The exponent, $\mathrm{n}$, for the $2 \mathrm{i}$ experiments reported by $\mathrm{S}$. $\mathrm{S}$. Stevens could be well approximated by the equation $n=1.53$ / $\log R_{\phi}$, and thus would range from approximately 6.1 to 0.8 for the span of ranges employed in the present loudness study. See Figure 4 in R. Teghtsoonian (1973).

3. See Note 6 for results of a test of this prediction.

4. This description is obviously based on values of $R_{y}$ no smaller than $5 \mathrm{~dB}$. It is conceivable that below that value the function may become nonlinear and define a different intercept, but that does not diminish the adequacy of Equation 5 for the case in which the intensities to be scaled exceed a range of $5 \mathrm{~dB}$, a case which describes virtually all scaling studies.

5. It should be noted that a similar result has been obtained in a two-stimulus loudness scaling experiment reported by Stevens and Poulton (1956). See Figure 9 in R. Teghtsoonian (1973) for the analysis of their data.

6. At the suggestion of an editorial referee, a miniature loudness scaling experiment was performed to see if the crossover between estimation and production exponents implied in Figure $2 \mathrm{~A}$ would occur if very small ranges were employed. The same loudness scaling procedures were used as before for two new groups of 16 subjects each, one using magnitude estimation and the other using magnitude production. In each case, the full span of six 
stimuli covered $.125 \mathrm{log}$ units, the actual selection of stimuli being determined as before. The median exponent for magnitude estimation was .62, and for magnitude production, it was .33 . A Mann-Whitney $U$ test showed this difference to be reliable $(\mathrm{U}=62, \mathrm{p}<.05$, two-tailed test). There are special difficulties associated with the use of such short ranges, but despite them, the results of this experiment support the view that the usual statement of the Stevens-Greenbaum regression effect. asserting that production always yields a larger exponent than estimation, is not tenable.

7. The complete derivation, in the form of an unpublished manuscript, is available from the first author on request.

Received for publication August 25, 1977, revision accepted July 18,1978 .) 